\section{Facharztprüfung zur Erlangung des Facharzttitels FMH für Physikalische Medizin und Rehabilitation}

Aufgrund des Weiterbildungsprogrammes, welches am 1. Januar 2000 in Kraft gesetzt wurde, ist die Teilnahme an der Facharztprüfung Voraussetzung für die Erteilung des Facharzttitels FMH für Physikalische Medizin und Rehabilitation. Es empfiehlt sich, die Facharztprüfung frühestens im letzten Jahr der reglementarischen Weiterbildung abzulegen (Art. 22 WB0).
Mündliche Prüfung (2. Teil)

Zulassungsbedingungen: Bestandene 1. Teilprüfung (European-Board-Facharztexamen).

Prüfungsort: Klinik St. Anna, Physikalische Therapie, Luzern

Datum: Dienstag, 4. Juni 2002

Prüfungsgebühr: Fr. 500.-

Schriftliche Anmeldung:

Sekretariat SGPMR c/o Schweiz. Rheumaliga,

Renggerstrasse 71, 8038 Zürich,

Tel. 0148740 00, Fax 0148740 19,

E-Mail: srl@srl.ch

Anmeldefrist: 30. März 2002

\section{Examen de spécialiste en vue de l'obtention du titre de spécialiste FMH en médecine physique et réadaptation}

Conformément au programme de formation postgraduée entré en vigueur le $1^{\mathrm{er}}$ janvier 2000, la participation à l'examen de spécialiste est obligatoire pour les candidats à l'obtention du titre de spécialiste FMH en médecine physique et réadaptation. Il est recommandé de passer l'examen de spécialiste au plus tôt durant la dernière année de formation postgraduée réglementaire (art. 22 RFP).

\section{Examen oral (2 partie)}

Conditions d'admission: Réussite de la première partie de l'examen (examen écrit de l'European Board).

Lieu d'examen: Klinik St. Anna, Physikalische Therapie, Lucerne

Date d'examen: mardi, le 4 juin 2002

Taxe d'examen: Fr. 500.-

Inscription écrite:

Secrétariat SSMPR

c/o Ligue suisse de rhumatologie,

Renggerstrasse 71, 8038 Zurich,

tél. 0148740 00, fax 0148740 19,

e-mail: srl@srl.ch

Délai d'inscription: le 30 mars 2002 